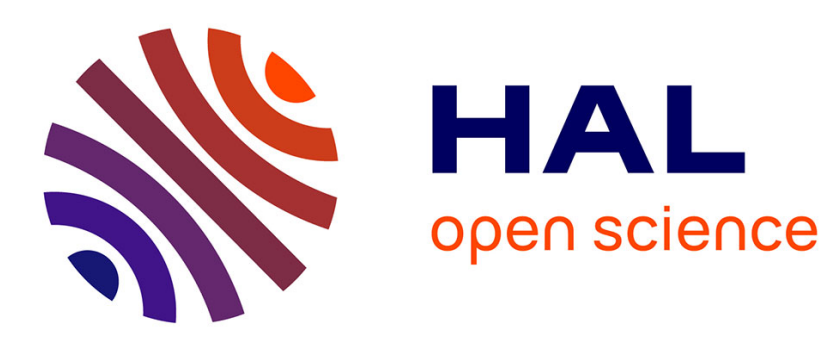

\title{
Contrail microphysics in the near wake of a realistic wing through RANS simulations
}

Florent Guignery, Emmanuel Montreuil, Olivier Thual, Xavier Vancassel

\section{To cite this version:}

Florent Guignery, Emmanuel Montreuil, Olivier Thual, Xavier Vancassel. Contrail microphysics in the near wake of a realistic wing through RANS simulations. Aerospace Science and Technology, 2012, vol. 23, pp. 399-408. 10.1016/j.ast.2011.09.011 . hal-00960260

\section{HAL Id: hal-00960260 \\ https://hal.science/hal-00960260}

Submitted on 17 Mar 2014

HAL is a multi-disciplinary open access archive for the deposit and dissemination of scientific research documents, whether they are published or not. The documents may come from teaching and research institutions in France or abroad, or from public or private research centers.
L'archive ouverte pluridisciplinaire HAL, est destinée au dépôt et à la diffusion de documents scientifiques de niveau recherche, publiés ou non, émanant des établissements d'enseignement et de recherche français ou étrangers, des laboratoires publics ou privés. 


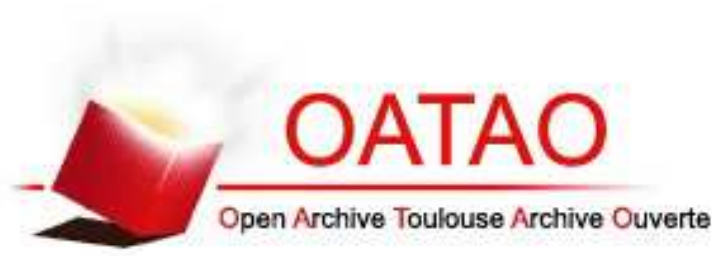

\section{Open Archive Toulouse Archive Ouverte (OATAO)}

OATAO is an open access repository that collects the work of Toulouse researchers and makes it freely available over the web where possible.

This is an author-deposited version published in: http://oatao.univ-toulouse.fr/ Eprints ID: 6706

To link to this article: http://dx.doi.org/10.1016/j.ast.2011.09.011

URL: http://www.sciencedirect.com/science/article/pii/S1270963811001611

\section{To cite this version:}

Guignery, Florent and Montreuil, Emmanuel and Thual, Olivier and Vancassel, Xavier Contrail microphysics in the near wake of a realistic wing through RANS simulations. ( In Press: 2011) Aerospace Science and Technology . ISSN 1270-9638

Any correspondence concerning this service should be sent to the repository administrator: staff-oatao@,listes.diff.inp-toulouse.fr 


\title{
Contrail microphysics in the near wake of a realistic wing through RANS simulations
}

\author{
F. Guignery ${ }^{1}$, E. Montreuil ${ }^{1}$, O. Thual ${ }^{2}$, X. Vancassel $^{1}$ \\ ${ }^{1}$ ONERA, DMPH/ENV ; 29 avenue de la Division Leclerc, F-93320 Chatillon, France \\ ${ }^{2}$ Université de Toulouse ; INPT ; CNRS ; IMFT; Allée Camille Soula, F-31400 Toulouse, France \\ 16/09/2011, revised version for "Aerospace Science and Technology"
}

\begin{abstract}
This paper focuses on Steady Reynolds Average Navier Stokes simulations (RANS) of ice particles growth in the near field of a wing-injector configuration. The multiphysics multiphase flow solver CEDRE, enriched with a microphysical model, has been developed in order to simulate the impact of a more real aircraft geometry in contrail formation studies. As a first evaluation case, a simplified aircraft description, i.e. a NACA0012 2D wing with two injectors, has been used. Ice formation has been simulated by assuming water condensation and instantaneous freezing on activated soot particles, initially emitted by aircraft engines. Our investigation focuses on the near field, extending from the nozzle exit until eight wing spans. Although the main goal is to address the question of ice formation, the aerodynamic flow field has been investigated and numerical results compared with existing experimental data. The first results indicate that the exhaust jet is correctly wrapped around the vortex and that the pattern of dilution qualitatively matches observations in the near field. Sensitivity studies to humidity and to the initial soot particle radius have also been performed.
\end{abstract}

\section{Introduction}

Aviation has known a rapid growth over the last fifty years. Passengers' traffic has increased by near 9 per cent per year since 1960 ([1]), and recent reports foresee the doubling of air traffic within fifteen years. In spite of constant technology improvement and subsequent engine emission indices reduction, aircraft total emissions keep increasing due to the faster growth of aviation transport demand. At cruising altitude the emitted gases as well as particulate matter modify the composition of the atmosphere. They contribute to the Earth's radiative balance perturbation by increasing the concentration of greenhouse gases, by forming contrails and potentially contrail-induced cirrus clouds for which a need for further research has been identified. Amongst key topics, particles resulting from incomplete combustion, mainly soot, provide ice nucleation sites and contribute therefore to the formation of condensation trails (contrails), artificially increasing cloudiness and 
altering climate ([1]). A lot of uncertainties concerning the predictive evaluation of this climate alteration exist ([2], [3]). To correctly assess the aircraft radiative impact due to contrails and induced cirrus, it is necessary to understand contrail formation and evolution processes on the different time and space scales involved. The lifetime of a contrail can reach several hours and its horizontal extent can be as large as several hundred kilometres. In the near field, contrail formation occurs during the exhaust jet and the marginal vortex interaction (see Figure 1).

The interaction between the engine jets and the trailing vortex is a basic step to be considered in order to understand how the exhausts mix with ambient air during the first seconds after emission and which role plays the vortex wake in this process ([4]). From the aerodynamics point of view, Miake-Lye et al. ([5]) identified two distinct phases. During the first few seconds after emission, the jets rapidly mix with ambient air (jet regime) while the vorticity moves from the wings and rolls up into a pair of trailing vortices. Later on, dynamics is dominated by the entrainment of the jets into the vortex flow.

Several experimental studies on aircraft wakes can be found in the literature. To characterize the dynamics of the jet in the vortex wake, Jacquin et al., ([6]) studied the flow downstream a scaled model, representative of a large transport aircraft in cruise conditions, using a Laser Doppler Velocimeter (LDV) system. The laser light sheet visualization revealed that part of the jet was already entrained at a distance of only half a wingspan downstream. Other studies ([7], [8]) revealed that, due to the velocity field of the vortex wake, additional vortices appear around the jet and lead to a quicker velocity decay. Margaris et al. ([9]) evaluated the effect of a cold jet on a single trailing vortex for different ratios of jet-to-vortex strength and jet-to-vortex distance and showed that the jet turbulence is wrapped around the vortex and ingested in it faster with decreasing jet-to-vortex distance and increasing jet strength. The trailing vortex is diffused and its rotational velocity and vorticity levels are reduced when the jet is located close to the vortex.

Direct numerical simulations (DNS) and large eddy simulations (LES) are commonly used for jet vortex interaction studies like in [10] and [11]. Both studies reported temporal simulations, i.e. for which the computational box is convected downstream with the air-flow velocity. This is based on the assumption of locally parallel flow, which means that the gradients of the mean flow in the axial direction are neglected over the short distance corresponding to the axial dimension of the simulation domain. The instabilities developing in this way are therefore of convective nature, in accordance with the fact that the Kelvin-Helmoltz instability developing in jet flows has been shown to be of such nature. Furthermore, these simulations are split into two sequential phases that correspond to the two regimes identified by [5]. This assumption is not straightforward looking at the 
complex flow behind an aircraft. Finally, these DNS and LES computations ignore the flow around the wing and initialize the tangential velocity profile of the vortex with a Lamb-Oseen model ([12]) and the jet with a hyperbolic tangential nozzle-exit profile.

To simulate the flow field around the wing, Fares et al. ([13]) proposed a new two step approach. First the flow field around the wing is determined; the data of this simulation are subsequently used to provide the inflow condition for a separate wake simulation to $1 / 2$ span. A steady Navier-Stokes for a compressible ideal gas solver was used. Due to the limited domain downstream of the wing $(1 / 2$ span), the region of entrainment was not reached. Two issues were shown to be crucial: the resolution of the vortex core and the accurate prediction of turbulence.

From a microphysical point of view, important physical and chemical processes take place in the plume and modify the initial properties of the emitted material. Engines emit gaseous compounds such as carbon dioxide $\left(\mathrm{CO}_{2}\right)$, water vapour $\left(\mathrm{H}_{2} \mathrm{O}\right)$, nitrogen oxides $\left(\mathrm{NO}_{\mathrm{x}}\right)$, sulphur oxides $\left(\mathrm{SO}_{\mathrm{x}}\right)$, carbon oxide ( $\mathrm{CO}$ ) and hydrocarbons. They interact together and form new species mainly through oxidation mechanisms. Sulphur dioxide is indeed oxidized into sulphuric acid which undergoes further transformation processes like phase transition as the plume cools down by entrainment of cold ambient air. Water vapour, sulphuric acid, chemi-ions and organics form a nucleating mixture, interact also with soot particles and finally lead to the formation of subvisible (submicronic aerosols) or visible (contrails) aerosols. The main criterion for contrail persistence is the supersaturation of the plume i.e. the plume water vapour has to be larger than the saturation vapour pressure above ice.

The numerical simulation of all these complex chemical and physical processes coupled with the resolution of the wake aerodynamics in one pass is computationally demanding and the cost can be prohibitive when too many details are required. Trajectory box models can be used (e.g.[14]) to describe microphysics occurring during the dilution of aircraft plumes at cruise altitude. However, no spatial information can be provided and the plume dilution is generally parameterised. This gap can be filled when simplified microphysics is implemented in fluid flow solvers ([15]). Paoli et al. ([16]) also presented an alternative approach to avoid this oversimplification on dynamical and mixing processes. Detailed offline microphysical processes computations were applied to an ensemble of fluid parcels trajectories generated by a LES two-phase flow solver. Interesting aerosol particles behaviour was shown, especially concerning the effect of the non-homogeneous mixing. However the coupling remained a one-way coupling where microphysics does not impact fluid properties. Finally, detailed aerodynamics simulations can be performed with online microphysics but it is generally limited to bulk ice microphysics and it does not uses real aircraft configurations. 
The present work focuses on the simulation of the growth of ice particles in the wake of a scaled model, the same as the one used during the aforementioned experimental study ([6]). The main goal is eventually to address the question of the airframe geometry impact on engine jet mixing and contrail properties as dilution is an important parameter. While numerous studies deal with the generation of vortices during plane take off or landing ([17]) for which the flow can be considered as incompressible, the present study deals with cruising flight for which the Mach number can be close to unity. The compressible multiphysics code CEDRE has thus been used ([18]), as it provides a large variety of solvers and openings towards further applications. The present solver deals with multispecies flows and uses a finite volume approach. Concerning the choice of the turbulence model, several authors have pointed out the need of a LES rather than a RANS approach which induced an excess of energy dissipation for the vortices ([19]). However, a RANS turbulence modelling is used since the focus is made here on the vortices generation in the presence of a complex wing geometry, which induces unstructured meshes and strong computational constraints. This flaw in vortices dissipation remains reasonable in the close wake of the wings.

\section{Model governing equations}

\subsection{Aerodynamics and species mixing}

The plume mixture has been assumed to be initially made of air, water vapour, and soot particles these being treated as ice crystals once favourable thermodynamic conditions are reached within the plume. Air and water vapour are considered as an ideal gas. The mass conservation equations for air density $\rho_{\text {air }}$, ice density $\rho_{\text {ice }}$ and water vapour density $\rho_{\text {vap }}$ are coupled to the 3D compressible Navier-Stokes equations for the mixing of density $\rho=\rho_{\text {air }}+\rho_{\text {ice }}+\rho_{\text {vap }}$. The system of equations reads in:

$$
\begin{gathered}
\frac{\partial}{\partial t}\left(\rho y_{k}\right)+\frac{\partial}{\partial x_{j}}\left(\rho u_{j} y_{k}\right)=\frac{\partial}{\partial x_{j}}\left(\rho D_{k} \frac{\partial y_{k}}{\partial x_{j}}\right)+\dot{w}_{k}, k \in\{\text { air, ice, vap }\} \\
\frac{\partial}{\partial t}\left(\rho u_{i}\right)+\frac{\partial}{\partial x_{j}}\left(\rho u_{i} u_{j}\right)=-\frac{\partial p}{\partial x_{i}}-\rho g \delta_{i 3}+\frac{\partial}{\partial x_{j}}\left(\mu S_{i j}^{d}\right), i=1, \ldots, 3, \\
\frac{\partial}{\partial t}\left(\rho e_{t}\right)+\frac{\partial}{\partial x_{j}}\left(\rho u_{j} h_{t}\right)=\frac{\partial}{\partial x_{j}}\left(\rho c_{p} \kappa \frac{\partial T}{\partial x_{j}}+\sum_{k} h_{k} D_{k} \frac{\partial y_{k}}{\partial x_{j}}+\mu S_{i j}^{d} u_{i}\right),
\end{gathered}
$$

for which the new variables are the velocity vector $\left(u_{1}, u_{2}, u_{3}\right)^{T}$, pressure $p$, total energy $e_{t}=e+\frac{1}{2} u_{i} u_{i}$ where $e$ is the internal energy, the total enthalpy $h_{t}=e_{t}+\frac{p}{\rho}$, temperature $T$ and 
the deviator strain rate tensor $S_{i j}^{d}=\left(\frac{\partial u_{i}}{\partial x_{j}}+\frac{\partial u_{j}}{\partial x_{i}}\right)-\frac{2}{3} \frac{\partial u_{l}}{\partial x_{l}} \delta_{i j}$ given by a Newton law with the Stokes hypothesis and the dynamical viscosity $\mu$. The three species $k \in\{$ air, ice, vap $\}$ are governed by their diffusion coefficient $D_{k}$ in the mixture and their mass transfer rate $\overline{\dot{w}}_{k}$. The thermal diffusivity is denoted by $\kappa$.

In the RANS approach, the equations are averaged so that any variable $\Phi$ may be decomposed into a mean part $\bar{\Phi}$ and a fluctuation part $\Phi^{\prime}$, with $\Phi=\bar{\Phi}+\Phi^{\prime}$. For compressible flows, a density weighted time average decomposition (also called Favre-average) is used: variables are then defined as $\tilde{\Phi}=\overline{\rho \Phi} / \bar{\rho}$ and $\Phi=\widetilde{\Phi}+\Phi^{\prime \prime}$. The new system of equations is then:

$$
\begin{gathered}
\frac{\partial}{\partial t}\left(\bar{\rho} \tilde{y}_{k}\right)+\frac{\partial}{\partial x_{j}}\left(\bar{\rho} \tilde{u}_{j} \tilde{y}_{k}\right)=\frac{\partial}{\partial x_{j}}\left(\bar{\rho} D_{k} \frac{\partial \tilde{y}_{k}}{\partial x_{j}}-\bar{\rho} \widetilde{u_{j}^{\prime \prime} y_{k}^{\prime \prime}}\right)+\bar{w}_{k}, k \in\{\text { air, ice, vap }\} \\
\frac{\partial}{\partial t}\left(\bar{\rho} \tilde{u}_{i}\right)+\frac{\partial}{\partial x_{j}}\left(\bar{\rho} \tilde{u}_{i} \tilde{u}_{j}\right)=-\frac{\partial \bar{p}}{\partial x_{i}}-\bar{\rho} g \delta_{i 3}+\frac{\partial}{\partial x_{j}}\left(\mu \tilde{S}_{i j}^{d}-\bar{\rho} \widetilde{u_{i}^{\prime \prime} u_{j}^{\prime \prime}}\right), i=1, \ldots, 3 \\
\frac{\partial}{\partial t}\left(\bar{\rho} \tilde{e}_{t}\right)+\frac{\partial}{\partial x_{j}}\left(\bar{\rho} \tilde{u}_{j} \tilde{h}_{t}\right)=\frac{\partial}{\partial x_{j}}\left(\bar{\rho} c_{p} \kappa \frac{\partial \bar{T}}{\partial x_{j}}-\bar{\rho} \widetilde{u_{j}^{\prime \prime} T^{\prime \prime}}+\sum_{k} \tilde{h}_{k} \bar{\rho} D_{k} \frac{\partial \tilde{y}_{k}}{\partial x_{j}}-\sum_{k} \tilde{h}_{k} \bar{\rho} \widetilde{u_{j}^{\prime \prime} y_{k}^{\prime \prime}}+2 \mu \tilde{S}_{i j}^{d} \tilde{u}_{i}-\bar{\rho} \widetilde{u_{i}^{\prime \prime} u_{j}^{\prime \prime} \tilde{u}_{i}}\right)
\end{gathered}
$$

The Reynolds tensor (i.e. $\widetilde{u_{i}^{\prime \prime} u_{j}^{\prime \prime}}$ ) is given by a Boussinesq hypothesis and the two equations k-I model. The turbulent diffusion fluxes of species and heat (respectively the terms $\widetilde{u_{j}^{\prime \prime} y_{k}^{\prime \prime}}$ and $\widetilde{u_{j}^{\prime \prime} T^{\prime \prime}}$ ) are evaluated by analogy with molecular diffusion flux:

$$
\begin{gathered}
\widetilde{u_{i}^{\prime \prime} u_{j}^{\prime \prime}}=-\nu^{t} \tilde{S}_{i j}^{d}+\frac{2}{3} k \delta_{i j}, \\
\widetilde{u_{j}^{\prime \prime T}}=-\kappa^{t} \frac{\partial \bar{T}}{\partial x_{j}}=-\frac{\nu^{t}}{\operatorname{Pr}^{t}} \frac{\partial \bar{T}}{\partial x_{j}}, \\
\widetilde{u_{j}^{\prime \prime} y_{k}^{\prime \prime}}=-D_{k}^{t} \frac{\partial \tilde{y}_{k}}{\partial x_{j}}=-\frac{\nu^{t}}{\operatorname{Sc}_{k}^{t}} \frac{\partial \tilde{y}_{k}}{\partial x_{j}},
\end{gathered}
$$

where the terms $\nu^{t}, \operatorname{Pr}^{t}$ and $\mathrm{Sc}_{k}^{t}$ correspond respectively to the turbulent eddy viscosity, the turbulent Prandtl number and the turbulent Schmidt numbers. The treatment of particles is detailed in the following section. 


\subsection{Microphysical processes}

The main assumption used in this work is that contrail formation is driven by ice heterogeneous nucleation on soot particles (e.g. [20]-[22]). Although all soot particles do not necessarily freeze, an upper limit case can be selected by first assuming that they are actually all activated. Therefore, all emitted soot particles may be considered as ice nuclei. They are assumed to be spherical which is a reasonable assumption according to the fact that ice crystals are mainly formed through a liquid solution freezing process, around a soot core [22], and that the turbulent plume does not promote any particular direction for water uptake.

The second hypothesis is that gas and particles (ice or soot) are in dynamical and thermal equilibrium. Soot particles are initially small, having mean sizes of the order of 30 to 50 nanometers and when contrails form, their size in the near field is about $1 \mu \mathrm{m}$ (e.g. [21]). The aerodynamic characteristic time $t_{c}$ for a particle of density $\rho^{p}$ and radius $r_{p}$ to be balanced with gas of dynamic viscosity $\mu$ is given by:

$$
t_{c}=\frac{\rho^{p} r_{p}^{2}}{72 \mu}
$$

For an ice particle with a diameter of one micrometer, this time is evaluated to $10^{-6}$ second. The characteristic time for a particle to be in thermal equilibrium with the gas is even less restrictive. We can then use an equivalent gas strategy for the treatment of particles.

Particles are followed through a passive scalar transport equation given by:

$$
\frac{\partial}{\partial t}(\bar{\rho} \tilde{Z})+\frac{\partial}{\partial x_{j}}\left(\bar{\rho} \tilde{u}_{j} \tilde{Z}\right)=\frac{\partial}{\partial x_{j}}\left(\bar{\rho} D_{Z} \frac{\partial \tilde{Z}}{\partial x_{j}}-\bar{\rho} \widetilde{u_{j}^{\prime \prime} Z^{\prime \prime}}\right)
$$

where $\tilde{Z}=\tilde{N}_{p} / \bar{\rho}, \tilde{N}_{p}$ represents the particles number density (number of particles per unit volume) and $D_{Z}$ is a the diffusion coefficient for the particle density. In the same way, the closure used for the turbulent diffusion $\widetilde{u_{j}^{\prime \prime} Z}$ is based on the turbulent eddy viscosity computed through the two equation $\mathrm{k}-\mathrm{I}$ model.

The mass density of ice in the plume can be worked out by a simple geometric argument and reads in:

$$
\bar{\rho}_{i c e}=\frac{4}{3} \pi\left(r_{p}^{3}-r_{s}^{3}\right) \bar{\rho}_{i c e}^{p} \tilde{N}_{p},
$$


where $\bar{\rho}_{i c e}^{p}$ is the mass density of ice in a particle which depends only on temperature. It can be taken from ([23]):

$$
\bar{\rho}_{i c e}^{p}(\bar{T})=0,916-8,75.10^{-5}(\bar{T}-273,15)-1,667 \cdot 10^{-7}(\bar{T}-273,15)^{2},
$$

where the variables are expressed in the international system of units (SI).

\subsection{Simplified condensation model}

A simplified transport model has been used for condensation of water vapour onto ice particles. Growth is evaluated using a modified Fick's law dedicated to mass transfer on particles whose radius $r_{p}$ is of the order of the mean free path $\lambda$. The mass transfer rate $\overline{\dot{w}}_{i c e}\left[\mathrm{~kg} \mathrm{~m}^{-3} \mathrm{~s}^{-1}\right]$, with $\overline{\dot{w}}_{i c e}=-\overline{\dot{w}}_{v a p}$, is given by

$$
\overline{\dot{w}}_{i c e}=\frac{4 \pi \tilde{N}_{p} r_{p} D_{v a p} M_{H_{2} O}}{R \bar{T}}\left(\bar{p}_{v a p}-\bar{p}_{v a p}^{\text {sat } / i c e}\right) G\left(r_{p}\right) \Pi\left(\bar{p}_{\text {vap }}^{\text {sat } / i c e}, r_{p}\right)
$$

where $M_{H_{2} \mathrm{O}}$ is the water molar mass, $R$ is the ideal gas constant. It depends on saturation conditions and more precisely on the difference between the water vapour partial pressure in the plume $\bar{p}_{\text {vap }}$ and the saturation vapour pressure above ice $\bar{p}_{\text {vap }}^{\text {sat } / i c e}$. This one is given by [24]:

$$
\ln \left(\bar{p}_{\text {vap }}^{\text {sat } / \text { ice }}\right)=9.550426-5723.265 / \bar{T}+3.53068 \ln \bar{T}-0.00728332 \bar{T},
$$

where the saturation vapour pressure and the temperature are expressed respectively in $\mathrm{Pa}$ and $\mathrm{K}$. The mass transfer rate is positive in condensation conditions $\left(\bar{p}_{v a p}>\bar{p}_{v a p}^{\text {sat } / i c e}\right)$, and negative when thermodynamic conditions are favourable to evaporation $\left(\bar{p}_{\text {vap }}<\bar{p}_{\text {vap }}^{\text {sat }}\right.$ ice $)$. It also depends on the particles number density $\tilde{N}_{p}$ and their radius $r_{p}$. The higher the particles number density, the larger the surface area is for condensation processes: the mass transfer rate $\overline{\dot{w}}_{i c e}$ increases accordingly to this rule.

The function $\Pi$ stops mass transfer when there is no more water on particles and is defined by:

$$
\Pi\left(\bar{p}_{\text {vap }}^{\text {sat } / \text { ice }}, r_{p}\right)=\left\{\begin{array}{l}
0 \text { when } \bar{p}_{\text {vap }} \leq \bar{p}_{\text {vap }}^{\text {sat } / \text { ice }} \text { and } r_{p}=r_{s} \\
1 \text { when } \bar{p}_{\text {vap }}>\bar{p}_{\text {vap }}^{\text {sat } / \text { ore }} \text { or } r_{p}>r_{s}
\end{array}\right. \text {. }
$$

The diffusion coefficient of water vapour in air $D_{\text {vap }}$ is given by [25] 


$$
D_{\text {vap }}=2.11 \times 10^{-5}\left(\frac{\bar{T}}{\bar{T}_{0}}\right)^{1.94}\left(\frac{\bar{p}_{0}}{\bar{p}}\right)\left[m^{2} / s\right] \text {, }
$$

with $\bar{T}_{0}=273.15 \mathrm{~K}$ and $\bar{p}_{0}=101325 \mathrm{~Pa}$. The function $G(r)$ takes into account the transition of the uptake from the gas kinetic to diffusion regime near the nanometric particle through the Knudsen number $K n=\lambda / r_{p}$ (see Figure 2). The Knudsen number is used to determine whether statistical mechanics or the continuum mechanics formulation of fluid dynamics should be used. If the Knudsen number is near or greater than one, the mean free path of a molecule is comparable to the particle size and the continuum assumption of fluid mechanics is no longer a good approximation. In this case, statistical methods must be used. Few formulations for the function $G\left(r_{p}\right)$ exist in the literature. Amongst them, [26] proposed

$$
G\left(r_{p}\right)=\left(\frac{1}{1+K n\left(r_{p}\right)}+\frac{4 K n\left(r_{p}\right)}{3 \alpha}\right)^{-1}
$$

where $\alpha$ is the deposition coefficient of water molecules on ice. We use the value proposed by [22], i.e. $\alpha=0.1$. The function $G$ takes the limiting values $G \rightarrow 1$ for $K n \rightarrow 0$ (diffusion limit) and $G \rightarrow 3 \alpha /(4 K n)$ for $K n \gg 1$ (free molecular regime).

Figure 3 represents the theoretical evolution of particles radius for a fixed supersaturation $\bar{p}_{\text {vap }} / \bar{p}_{\text {vap }}^{\text {sat } / \text { ice }}=3.11$ and different initial Knudsen number $K n_{0}=\lambda / r_{p_{0}}$ where $r_{p_{0}}$ is the initial radius, from [22]. We notice that the smaller is the mean free path $\lambda$, the higher is the particles growth rate at fixed $r_{p_{0}}$. The final particles size is not affected since it only depends on equilibrium thermodynamics conditions.

\section{Numerical simulations}

\subsection{From wind tunnel experiments to cruising altitude simulations}

A first series of numerical simulations is based on a wind tunnel experiment ([6]). This experiment studied the flow field produced by the interaction of two heated jets with the wake vortices of a 2D NACA0012 wing profile (see Figure 4). The characteristic dimensions of the model were based on similarity parameters of a large transport aircraft with two engine jets. This experimental study, detailed in [6], delivered a database on the flow until eight spans behind the setup, which has been used for validating our numerical tool from an aerodynamic point of view. It was checked that the 
supporting structure of the wing (see Figure 4) induced only weak perturbations on the observed phenomena. The experimental conditions are given in Table 1. The experimental results include mean and fluctuating velocity fields (measured with a LDV system) and mean temperature field (measured with thermocouples).

Thus, parameters were set in the model so as to reproduce the experiment in the wind tunnel. The velocity, temperature and pressure of the external flow are $\bar{U}_{a}=20 \mathrm{~m} \mathrm{~s}^{-1}, \bar{T}_{a}=293 \mathrm{~K}$ and $\bar{p}_{a}=101325 \mathrm{~Pa}$ respectively. The jet velocity has been initialised to $\bar{U}_{J}=60 \mathrm{~m} \mathrm{~s}^{-1}$ and its temperature at $\bar{T}_{J}=583 \mathrm{~K}$. The boundary conditions are summarized in Table 2 . The initial jet vortex separation has been set to $d_{J v}=0.21 b$. Thus, the marginal vortex is initially at a distance of 17 vortex core radii $r_{c}$ from the jet centre. Buoyancy effects, which are taken into account in the model, are small according to the low value (0.025) of the ratio $\frac{1}{F r^{2}}=\frac{g b}{U_{a}}$.

A second series of simulations is then conducted in order to have the same thermodynamic conditions as in the atmosphere at cruising altitude, enabling ice formation. Temperature and pressure of the external flow have been thus set to $\bar{T}_{a}=220 \mathrm{~K}$ and $\bar{p}_{a}=22000 \mathrm{~Pa}$. The external flow has been considered as being made of air and water vapour. The ambient relative humidity was $S_{\text {liq }}=\bar{p}_{\text {vap }} / \bar{p}_{\text {vap }}^{\text {sat } / l i q}=0.6$ that is corresponding to a water vapour saturation ratio of $S_{\text {ice }}=\bar{p}_{\text {vap }} / \bar{p}_{\text {vap }}^{\text {sat } / i c e}=0.98$ with respect to ice. The injectors are supposed to release water vapour into the atmosphere with an emission index $E I_{\mathrm{H}_{2} \mathrm{O}}=1.15 \mathrm{~kg}_{\mathrm{H}_{2} \mathrm{O}}$ per $\mathrm{kg}_{\text {fuel }}$ (typical for a Rolls-Royce RB211 engines for Boeing 767 for instance). The emission index of a species corresponds to the mass of this species generated by the burn of one kilogram of fuel. The relation between water emission index and water vapour partial pressure is given by

$$
\bar{p}_{v a p}^{\text {exit }}=\frac{E I_{\mathrm{H}_{2} \mathrm{O}}}{(A F R+1)} \frac{M_{a i r}}{M_{\mathrm{H}_{2} \mathrm{O}}} \bar{p}_{a},
$$

where $M_{\text {air }}$ is the air molar mass and $A F R=60$ is the air to fuel ratio that has been chosen. The water vapour partial pressure at the exit of the jet was thus, $\bar{p}_{v a p}^{e x i t}=660 \mathrm{~Pa}$. The injectors were also supposed to emit soot particles. Their emission index was $E I P_{s}=3.510^{14} \mathrm{~kg}_{\text {fuel }}^{-1}$. This value is proper 
to a commercial transport aircraft ([27]). The number density of engine emitted soot particles $\tilde{N}_{p}^{e x i t}$ is linked to their emission index through the relation:

$$
\tilde{N}_{p}^{e x i t}=\frac{E I P_{p} \bar{\rho}_{\text {air }}}{A F R+1}=8.1 \leftrightarrow 10^{11} \mathrm{~m}^{-3}
$$

Their initial radius was $r_{p}=r_{s}=10 \mathrm{~nm}$. The distribution is assumed to be mono-dispersed for this first modelling attempt. Entrainment of ambient aerosols in the plume has not been considered in our study, since we focus on the formation processes in the near field of the aircraft, at about 1-2s behind the engine. In this area, the particles number density is still dominated by engine emissions, which are several orders of magnitude higher than ambient aerosols. These may play a role when the plume gets diluted enough so that engine emitted and ambient particles have concentrations of the same order of magnitude (see for instance [28]). Our conclusions on the formation processes remain therefore valid. It should also be noted that ambient aerosols are often neglected in detailed microphysics calculations (e.g. [29]).

\subsection{Numerical method and mesh generation}

The numerical code CEDRE used for this study is a parallel, three-dimensional, multi species and compressible Navier-Stokes solver. The numerical method is based on a cell centred finite volume approach for general unstructured grids. It has been developed at ONERA and was successfully used for predicting a wide variety of flow phenomena including aerodynamics and combustion (see [30][32]). The spatial discretization is performed by using a second order scheme based on the MUSCL approach ([33]) for both convective and viscous terms. A RANS approach based on the k-I turbulence model is used. Only stationary states are computed using a pseudo-time evolution of the temporal equations with a first order linear-implicit Euler scheme. The implicit linear system resolution is done by a GMRES method. In this scheme, a scaling of the Jacobian matrix block associated to the water vapour and ice density fluxes is performed in order to avoid the convergence towards unstable microphysics states. Such states correspond to the natural supersaturation of the water vapour that is removed on very short time scales once soot particle are present.

The necessity to resolve a lot of space scales, from the characteristic length of the vortex $r_{c}=610^{-3} \mathrm{~m}$, for instance, to macroscopic scales such as the jet entrainment until eight spans $(8 b=4 \mathrm{~m})$, makes the mesh generation complex. The computational domain dimensions are $16 b \times 4 b \times 8 b$. A sketch of this domain is given in Figure 5. Near the wing, a prism layer is used to correctly resolve the shear layer (see Figure 6). At the wall, the height of the first cell is about 
$210^{-5} \mathrm{~m}$. Two cylindrical refinement areas with tetrahedral elements are centred on the vortex. In the wake, a structured hex block is inserted to minimize the numerical dissipation in the convection of the marginal vortex. The central part is modified in order to have homogeneous sized cells of $10^{-3} \mathrm{~m}$ in the vortex core in order to get 7 points in the vortex radius. The radius of this structured block is $120 \mathrm{~mm}$, i.e. the jet vortex separation. The size of the computational grid is 9732516 cells and 22654078 facets. All the numerical simulations have been carried out over 64 processors. For each 3D numerical simulation, the CPU time consuming per processor was about 120 hours.

\section{Results and discussion}

\subsection{Wind tunnel model comparison and jet vortex interaction}

At each wing tip, a rotational flow takes place. The marginal vortex results from a complex flow around the wing tip and the merging of multiple vortices from the tip and suction side. Figure 7 shows iso-contours of axial velocity in the region of the wing tip. We notice that the marginal vortex comes from the merging of mainly two vortices, one from the wing tip and another from the suction side. These are formed due to the leakage of flow from the wing surface towards the tip region. This is due to a strong spanwise pressure gradient that is present in this region. The pressure side vortex convects towards the suction side vortex and merges with it. At $x / c=1$, a dominant vortex is moving significantly inwards away from the tip region. By plotting the cross plane velocity vectors at several streamwise locations beyond the trailing edge, we notice as expected that the marginal vortex in the very near wake keeps moving inwards away from the tip region (not shown).

Iso-values of flow temperature are plotted in Figure 8 for different downstream cross sections (over eight spans). The entrainment process is shown as a stretching and distortion of the interface between the engine jet and the external flow. More precisely, the cross sections of dimensionless

temperature at $x / b=1, x / b=5$ and $x / b=8$ are presented in Figure 9. Results coming from the experiment and numerical simulations are plotted on the left hand side and on the right hand side respectively. Figure 9a shows a stretching and distortion of the interface between the jet and the external flow. Figure $9 b$ and Figure $9 c$ show that the qualitative features of the jet entrainment are relatively well predicted by the numerical simulation at eight wingspans. Nonetheless, the entrainment of the computed jet flow by tangential momentum is slightly delayed as seen on Figure 9c. It also noticeable when comparing numerical velocity profiles with LDV measurement (not shown here): the intensity of the numerical vortex decreases more rapidly than the experimental one. This 
delay was also found in a DNS simulation of a similar jet vortex interaction ([10]) and this flow is characteristic of RANS simulations ([19]).

Finally, Figure 10 presents the downstream variations of the peak values of the experimental and numerical normalized temperature. The numerical results show a good agreement with the experimental measurements.

\subsection{Ice particles growth during the jet vortex interaction}

All particles are initially under-saturated since they are still concentrated inside the hot jet region. Due to mixing with cold air the plume cools down, becomes supersaturated with respect to liquid water. Soot particles get activated and are assumed to freeze immediately. Saturation with respect to ice therefore controls the kinetic of condensation and evaporation processes.

Water vapour pressure $\bar{p}_{v a p}$ is strongly linked to the vapour mass density $\bar{\rho}_{\text {vap }}$ because of small pressure fluctuations. Just behind the aircraft, the jet is sub-saturated, despite the important water vapour amount available because of high temperature levels. The jet-mixing layer enables the decrease of temperature (and vapour mass fraction). The jet side-mixing layer becomes supersaturated and ice crystals appear. The jet is stretched by the marginal vortex to three spans and then rolled up. Ice crystals start growing when the temperature in the entire jet is low enough for the vapour partial pressure to be higher than the pressure at saturation $\left(\bar{p}_{\text {vap }}>\bar{p}_{\text {vap }}^{\text {sat } / \text { ice }}\right)$.

Figure 11 presents the three-dimensional spatial growth of ice crystals in the wake until eight spans. The iso-surface of the particle number density $\tilde{N}_{p}=510^{9} \mathrm{~m}^{-3}$ is colored with ice particles radius. The crystals whose radius is higher than ten nanometers (soot radius) are visible from half a span. The particles grow when moving away from the nozzle since temperature decreases. One notices a slight asymmetry between the two vortices, which traces back to the spatial growth of small perturbations in turbulent flows.

\subsection{Ice particles size distribution}

Figure 12a shows iso-contours of saturation with respect to ice $S_{i c e}$ and iso-lines of ice rate at three, five and eight spans. At three spans, the jet is already fully supersaturated and we notice that the saturation peak is not at the centre of the jet while this is the case at five or eight spans. Indeed, at three spans, even if the water vapour content peaks at the centre of the jet (most of the water in the 
wake comes from the engines), temperature is still higher (only a few Kelvin) than at the edges of the jet. On the other hand the jet temperature at eight spans is almost homogeneously distributed and the saturation peak only depends on water vapour fraction whose maximum is still at the jet centre. Iso-contours of particles number density are plotted in Figure $12 \mathrm{~b}$ for the three downstream cross sections. There is no interaction between particles and no formation of new ones. Particles are just convected and diffused in the shear layer between the jet and the external flow. Since all particles (soot) are emitted by the engines, the particles concentration peak stays at the centre of the jet that is gradually stretched and rolled up by the marginal vortex. At three, five and eight spans, all particles are saturated and enable water vapour deposition. The spatial growth of particles is not homogenous since the ice mass transfer mainly depends on saturation and particles number density (see Equation (1.14)). Iso-contours of particles radius are plotted in Figure $12 \mathrm{c}$ for the three downstream cross sections. At $x / b=8$, particles can reach a radius of $350 \mathrm{~nm}$. We notice that the maximum particles size is reached where mass water content and particles number density are at their maximum level as well.

The particles size distribution is given by Figure 13. The mean and the variance of the particle distribution increase with the distance and exhibit a strong asymmetry with a median smaller than the mean. These features can be related to the turbulent mixing. Mixing is more efficient farther away behind the trailing edge. We note that the maximum radius at eight spans is around $350 \mathrm{~nm}$. This value remains weak in comparison to in situ measured values. The time of residence of a particle in the wake can explain this observation. It corresponds to the time for a particle that has been emitted by the engine to reach eight spans. This time is around 2 seconds in the case of a realistic aircraft and only 0.2 second in the case of the experimental set-up. Provided that the environment is always supersaturated and that deposition characteristic times remain unchanged, a particle would grow to a larger size with increased residence time.

\subsection{Sensitivity to soot radius and ambient relative humidity}

The characteristic dimensions of the model were based on similarity parameters of a large transport aircraft with two engine jets, particularly for the Long number [34], which respects similarities from an aerodynamics point of view. This number is not fully appropriate to characterize a realistic jet vortex interaction when focusing on microphysics occurring in the wake.

Some aircraft features such as wing geometry, engine technology and position for instance may change contrail's characteristics and influence their radiative impact. It can be assessed through the 
optical depth $\tau$ that depends on particles surface area, particles concentration, contrail geometrical depth $l_{c}$ and an extinction coefficient $Q_{e x t}$ and reads in:

$$
\tau=\pi r_{p}^{2} Q_{e x t} \tilde{N}_{p} l_{c}
$$

Particles size distribution in a contrail has to be known to get this value, as well as the spatial distribution and the contrail dimensions. This one may strongly depends on aircraft features and atmospheric conditions.

Figure 14a represents the effect of the initial soot radius $r_{s}$ on the particles size distribution at eight spans. Since the number of particles is unchanged, the soot mass as $r_{s}^{3}$. Red lines correspond to $r_{s}=10 \mathrm{~nm}$ and green lines correspond to $r_{s}=100 \mathrm{~nm}$. We notice that, in the two cases, the shape of the distribution is quite the same. The distribution is only shifted towards larger radii when emitted soot particles are initially larger ([35]). At eight spans the distribution is centred on $220 \mathrm{~nm}$ with a mean radius around $210 \mathrm{~nm}$ when $r_{s}=10 \mathrm{~nm}$, while it is centred on $310 \mathrm{~nm}$ with a mean radius around $300 \mathrm{~nm}$ when $r_{s}=100 \mathrm{~nm}$.

Figure $14 \mathrm{~b}$ shows the effect of the atmospheric relative humidity $S_{l i q}=p_{v a p} / p_{v a p}^{\text {sat } / \text { liq }}$ on the ice crystals size distribution at eight spans. In the reference case, the atmospheric relative humidity is set to $S_{l i q}=0.6$ corresponding to $S_{i c e}=0.98$. A second case is presented where atmospheric conditions are more favourable to evaporation of ice crystals through the choice of $S_{l i q}=0.3$ and $S_{i c e}=0.49$. The particles size distribution is then centred on $200 \mathrm{~nm}$ against $220 \mathrm{~nm}$ in the reference case. The mean radius is also smaller since $r_{\text {mean }}=180 \mathrm{~nm}$ when $S_{l i q}=0.3$ and $r_{\text {mean }}=210 \mathrm{~nm}$ when $S_{l i q}=0.6$. The low atmospheric water vapour content limits the amount of condensable water vapour in the jet (due to entrainment processes) and enables ice crystals at the jet periphery to evaporate. Nevertheless, even if the atmosphere is ice sub-saturated, ice crystals grow up to eight spans. Indeed, in the near field of the aircraft contrail formation is mainly dominated by the jet properties (water vapour amount, soot concentration, etc.). Atmospheric conditions play a key role in the spreading of the contrail and its time evolution (ice crystals concentration and size).

\section{Conclusion}

Spatial CFD computations on a NACA0012 wing with injectors have been carried out using the ONERA multiphysics code CEDRE. This work has shown that the jet vortex interaction studies, taking into account a full complex geometry with the use of unstructured mesh, provides valuable insight of 
contrail formation. Although the rollup intensity could be improved, these first results point out that the dilution ratio based on the maximum temperature in the plume is fairly well described. To study ice formation for different wing/engine configurations (jet location, wing airfoil), developments have been integrated in CEDRE to enable condensation and evaporation on nanoparticles using explicit or implicit temporal scheme. This new modelling features led to the first results of microphysics phenomena occurring during a spatial jet vortex interaction. The ice-growth model, coupled to aerodynamics resolution has provided the size and spatial distribution of ice particles until eight spans downstream the injector setup. These first computations of microphysics in the wake of a downscaled setup have shown that the particle sizes at eight spans downstream from the emission are smaller than in the case of a realistic aircraft in cruise (a few micrometers). Nevertheless, this first study, whose aerodynamical fields have been compared to experimental data provided by the FARWAKE project, reveals that the code CEDRE is able to lead a complex computation of a spatial jet vortex interaction from both aerodynamical and microphysical point of views. Some key parameters on contrail formation, such as soot radius and atmospheric relative humidity, have been studied and their impact on the size and spatial distribution of ice crystals up to eight spans have been quantified. Further investigations are needed to better characterize the location where ice crystals appear downstream. Further work will then consist in implementing a new computation case in order to get a realistic size distribution of ice particles to eight spans behind a real aircraft in cruise conditions.

\section{References}

[1] IPCC, Aviation and the global atmosphere - A special report of IPCC working groups I and III. Intergovernmental Panel on Climate Change, Cambridge, UK and New York.1999

[2] R. Sausen, I. Isaksen, V. Grewe, D. Hauglustaine, D. S. Lee, G. Myhre, M. O. Köhler, G. Pitari, U. Schumann, F. Stordal, C. Zerefos, Aviation radiative forcing in 2000: An update on IPCC (1999), Meteorologische Zeitschrift, 14 (2005) 555-561.

[3] D. S. Lee, D. W. Fahey, P. M. Forster, P. J. Newton, R. C. N. Wit, L. L. Lim, B. Owen, R. Sausen, Aviation and global climate change in the 21st century, Atmospheric Environment, 43 (2009) 3520-3537.

[4] R. Paoli, F. Garnier, Interaction of exhaust jets and aircraft wake vortices: small-scale dynamics and potential microphysical-chemical transformations, Comptes Rendus Physique, 6 (2005) 525547.

[5] R. C. Miake-Lye, R. C. Brown, C. E. Kolb, Plume and wake dynamics, mixing and chemestry behind a high speed civil transport aircraft, J. Aircraft, 30 (1993) 467-479. 
[6] L. Jacquin, P. Molton, P. Loiret, E. Coustols, An Experiment on Jet-Wake Vortex Interaction, in 37th AIAA Fluid Dynamics Conference and Exhibit, Miami (FL), USA, 2007, 43-63.

[7] F. Y. Wang, M. M. J. Proot, J.-M. Charbonnier, Near-Field Interaction of a Jet with Leading-Edge Vortices, Journal of Aircraft, 37 (2000) 779-785.

[8] F. Y. Wang, K. M. Q. Zaman, Aerodynamics of a Jet in the Vortex Wake of a Wing, AIAA Journal, 40 (2002), 401-407.

[9] P. Margaris, D. Marles, I. Gursul, Experiments on jet/vortex interaction, Exp Fluids, 44 (2008) 261-278.

[10] C. F. Gago, S. Brunet, F. Garnier, Numerical investigation of turbulent mixing in a jet/vortex interaction, AIAA Journal, 40 (2002) 276-284.

[11] R. Paoli, F. Laporte, B. Cuenot, T. Poinsot, Dynamics and mixing in jet/vortex interactions, Physics of fluids, 15 (2003) 1843-1860.

[12] H. Lamb, Hydrodynamics. New York, 1923.

[13] E. Fares, M. Meinke, W. Schröder, Numerical Simulation of the interaction of Wingtip Vortices and Engine Jets in the Near Field, in AIAA, DENVER, CO, 2000.

[14] B. Kärcher, A trajectory box model for aircraft exhaust plumes, Journal of Geophysical Research, 100 (1995) 18835-18844.

[15] J. Wu, S. Menon, Aerosol dynamics in the near field of engine exhaust plumes, Journal of Applied Meteorology, 40 (2001) 795-809.

[16] R. Paoli, X. Vancassel, F. Garrnier, P. Mirabel, Large-eddy simulation of a turbulent jet and a vortex sheet interaction: particle formation and evolution in the near field of an aircraft wake, Meteorologische Zeitschrift, 17 (2008) 131-144.

[17] T. Gerz, Holzäpfel, F., Bryant, W., Köpp, F., Frech, M., Tafferner, A., Winckelmans, G. Research towards a wake-vortex advisory system for optimal aircraft spacing, Comptes Rendus Physique, Special issue on Aircraft trailing vortices, 6 (2005) 501-523.

[18] P. Chevalier. et al., CEDRE: development and validation of a multiphysic computational software. (pdf 3,59 Mo), 1st European Conference for Aerospace Sciences (EUCASS), Moscou, Russia, 2005.

[19] Lewellen and Lewellen, The Effects of Aircraft Wake Dynamics on Contrail Development, J. Atmo. Sci, 58 (2001), 390-406

[20] Kärcher B., Physicochemistry of aircraft-generated liquid aerosols, soot, and ice particles. 1. Model description, J. Geophys. Res., 103 (1998) 17111-17128

[21] Kärcher B. et al., Physicochemistry of aircraft-generated liquid aerosols, soot, and ice particles. 2. Comparison with observations and sensitivity studies, J. Geophys. Res., 103 (1998) 17129-17148.

[22] B. Kärcher, The initial Composition of Jet Condensation Trails, Journal of the atmospheric sciences, 53 (1996) 3066-3083. 
[23] A. Tabazadeh, S. T. Martin, J. S. Lin, The effect of particle size and nitric acid uptake on the homogeneous freezing of aqueous sulphuric acid particles, Geophys. Res. Let., 27 (2000) 11111114.

[24] D. M. Murphy, T. Koop, Review of the vapour pressures of ice and supercooled water for atmospheric applications. Q. J. R. Meteorol. Soc. 131 (2005) 1539-1565.

[25] W. D. Hall, H. R. Pruppacher, The survival of ice particles falling from cirrus clouds in subsaturated air, J. Atmos. Sci., 33 (1976) 1995-2006.

[26] Y. S. Sedunov, Physics of Drop Formation in the Atmosphere, J. Wiley, Ed., ed, 1974, p. 234.

[27] F. Garnier, C. Baudoin, P. Woods, N. Louisnard, Engine emission alteration in the near field of an aircraft, Atmospheric Environment, 31 (1997) 1767-1781.

[28] Kärcher B. and F. Yu, Role of aircraft soot emissions in contrail formation. Geophys. Res. Lett. 36:L01804 (2009) doi:10.1029/2008GL036694.

[29] Wong H.-W., R. C. Miake-Lye, Parametric studies of contrail ice particle formation in jet regime using one-dimensional microphysical modelling, Atmos. Chem. Phys., 10 (2010), 3261-3272.

[30] A. Murrone, D. Scherrer, Large Eddy Simulation of a turbulent premixed flame stabilized by a backward facing step, in 1st INCA Workshop, Villaroche, France, 2005.

[31] L. Tesse, S. Avila, F. Dupoirieux, J. Taine, Coupled modeling of aerothermochemistry, soot formation and radiation, in a turbulent diffusion flame, in 13rd International Heat Transfer Conference, Sydney, Australie, 2006.

[32] M. Huet, F. Vuillot, G. Rahier, Numerical study of the influence of temperature and micro-jets on subsonic jet noise, in 14th AIAA/CEAS, Aeroacoustics Conference, Vancouver, Canada, 2008.

[33] B. Van Leer, Toward the Ultimate Conservative Difference Scheme V. A Second-Order Journal of Computational Physics, 32 (1979) 101-136.

[34] R. R. Long, A vortex in an infinite viscous fluid, J. Fluid Mech., 11 (1961) 611-626.

[35] U. Lohmann, J. Feichter, Global indirect aerosol effects: a review, Atmospheric Chemistry and Physics, 5 (2005) 715-737. 